\section{Suspected transmission of mycobacterium xenopi to a domestic albino ferret from exposure to infected aquarium water}

\author{
Natasha Davendralingam², \\ Nadene Stapleton ${ }^{1}$, Vicki Baldrey ${ }^{1}$, \\ Mark Stidworthy ${ }^{4}$, Laureen M Peters ${ }^{1}$, \\ Indran Davagnanam ${ }^{3}$
}

1 Beaumont Sainsbury Animal Hospital, Royal Veterinary College, London, UK

2 St Bartholomews Hospital, London, UK

3 National Hospital of Neurology and Neurosurgery (Queen Square), London, UK

4 International Zoo Veterinary Group Pathology, West Yorkshire, UK

\section{INTRODUCTION}

Mycobacterium xenopi more commonly infects reptiles, amphibians and marine life with water as its proven reservoir. It is very rare in ferrets. As the affected ferret was bred and kept in captivity, the infection would have been contracted from an external/environmental source.

\section{OBJECTIVES}

To identify the source of mycobacterium xenopi infection in a domestic albino ferret i.e. animal-animal, human-animal or environmentally-derived.

\section{METHODS}

1. Affected ferret: Presented with a 2-month history of weight loss, anorexia and malaise. The ferret underwent a clinical examination, x-rays, blood tests and lymph node biopsy. Due to poor prognosis and rapid deterioration, the ferret was euthanised. A post-mortem examination was conducted

2. Human contacts: The primary owners underwent full medical assessments with respiratory specialists. A detailed survey of 10 humans with recent contact with affected ferret was conducted

3. Companion ferrets: 4 ferrets sharing living-quarters underwent full veterinary assessment

4. Water analysis: Samples from aquarium sent for analysis (The affected ferret was the sole ferret with contact with water from this aquarium.)

\section{RESULTS}

1. Mycobacterium xenopi present in lungs (primarily), liver and spleen

2. No identifiable risk of human-ferret transmission

3. None affected

4. Awaiting sub-type confirmation from submitted samples

\section{CONCLUSIONS}

High suspicion of mycobacterium xenopi transmission through consumption/inhalation of infected aquarium water.

This is a unique collaboration between medical $\&$ veterinary doctors and pathologists to investigate a cause for infection which concluded with the rare (second ever recorded case) contraction of mycobacterium xenopi in a ferret through an unconventional mode of transmission; aquarium water.

No funding or declarations of interest to disclose.

\section{Aortic embolisation in two cats; is it always a matter of the heart?}

\author{
Lamprini Kalogianni ${ }^{1}$, Dimitris Timiou ${ }^{1}$, \\ Theodora K. Tsouloufi ${ }^{1}$, \\ Nektarios Soubasis ${ }^{1}$, Michail Patsikas ${ }^{1}$, \\ loannis Panopoulos', \\ Konstantina Theodorou ${ }^{1}$, Dimitra Psalla ${ }^{1}$, \\ Lina Tentoma ${ }^{3}$
}

1 School of Veterinary Medicine, Faculty of Health Sciences, Aristotle University of Thessaloniki, Thessaloniki, Greece

2 Private Practitioner, Athens, Greece

3 Private Practitioner, Thessaloniki, Greece

\section{OBJECTIVES}

To describe two feline cases with aortic embolisation and subsequent hindlimb paralysis due to a primary lung tumor.

\section{METHODS}

Cat 1 and Cat 2 were referred within four years. Both were domestic shorthairs, 12 and 15 years old, with lower motor neuron signs in both hindlimbs, bilateral absence of femoral pulses, hypothermia and cyanotic paw pads. Cat 1 had a $3 \mathrm{~d}$ grade heart murmur. Peripheral affected 